\title{
Scanning Tunneling Potentiometry of Semiconductor Junctions
}

\author{
Yang Dong and R. M. Feenstra \\ Dept. Physics, Carnegie Mellon University, Pittsburgh, PA 15213 \\ R. Hey and K. H. Ploog \\ Paul-Drude-Institut für Festkörperelektronik, Hausvogteiplatz 5-7, D-10117, Berlin, \\ Germany
}

\begin{abstract}
A method for performing scanning tunneling potentiometry of semiconductor heterojunctions is described. The method yields a direct measure of the electrostatic potential distribution across the interface, with microscopic resolution. The measurement is accomplished by scanning the probe tip at constant sample-tip separation across the junction, and adjusting the sample-tip voltage to maintain a constant tunnel current. An example is given of potentiometry across a GaAs pnjunction.
\end{abstract}

\section{Introduction}

Heterostructure devices have widespread application in solid state electronics. Valence and conduction band discontinuities, and the built-in potentials determine the performance of these devices. Determining those parameters would have an obvious impact on the optimization of devices. There exist many methods for such measurements including transport methods, optical techniques, internal photoemission and photoemission spectroscopy. The main disadvantage of those methods is the lack of good spatial resolution in the direction perpendicular to the interface. However, scanning tunneling microscopy (STM) performed in a cross-sectional manner on a cleaved surface permits the study of buried interfaces with high spatial resolution. STM has been previously used for potentiometric measurement of both metallic and semiconducting [1-6] materials. In the latter case however, the prior work does not provide a direct measurement of the electrostatic potential, as discussed in more detail below. In this work, we provide a new method to obtain in a direct, parameter-free way the electrostatic potential versus position in a semiconductor heterojunction. A demonstration of the method is provided by measurements across GaAs pn-junctions.

\section{Experimental}

The sample studied here is pictured in Fig. 1. Two GaAs pn-junctions and one GaAs/AlAs/GaAs heterostructure were grown on an $n$-type $\mathrm{GaAs}(001)$ substrate using molecular beam epitaxy. A (1 $1 \overline{1} 0)$ surface was exposed by cleavage in the STM chamber, under a background pressure of less than $4 \times 10^{-11}$ Torr. Commercially available Pt-Ir probe tips were used in the STM. Field-emission was used to assess the probe-tip sharpness; the tips typically require voltages of -400 to $-500 \mathrm{~V}$ in order to achieve an emission current of $10 \mathrm{pA}$, from which we deduce tip radii in the range $20-40 \mathrm{~nm}[7,8]$. Topographic imaging was performed at a constant current of $0.1 \mathrm{nA}$ and at sample-tip voltages specified below. To minimize the effects of tip-induced band bending, a $10 \mathrm{~mW}$ He-Ne laser focused to a spot size of about $3 \mathrm{~mm}$ was directed onto the cleaved surface, making a glancing incidence of about $5^{\circ}$ to the surface. This illumination was however found to have little effect on the results for the potentiometric scans (perhaps because it was not intense enough), as discussed 
below.

\section{Results}

A large-scale STM image of the sample we have studied is shown in Fig. 1. The $n$-type substrate is seen on the right-hand side of the image, and to the left of that are seen the two GaAs $p n$-junctions. The depletion layers of the $p n$-junctions can be clearly identified in the STM image. Due to a lack of carriers in the depletion layers those regions appear dark in comparison with regions far from the junction. The observed contrast is purely an electronic effect since the cleavage face is atomically flat. Typical current-voltage $I(V)$ curves from the $n$ - and $p$-type layers of the lowdoped $p n$-junction are shown in the bottom panel of Fig. 1. As observed in prior works $[6,9,10]$, the $I(V)$ curves display the band-gap of GaAs, and the Fermi-level $(0$ V) shifts across the gap as one moves from $n$ - to $p$-type material. By performing spatially resolved $I(V)$ measurement across a $p n$-junction, and analyzing these $I(V)$ curves to obtain a band edge location, one can of course directly map out the variation in band edge location across a junction. This technique was successfully applied by Yu et al. in their study of Si pn-junctions [6]. In contrast, the potentiometric technique proposed here involves imaging at voltages well above the conduction band edge in order to directly measure the potential variation in a junction.

The basic principle of our method is illustrated in Fig. 2, using a pn-junction as an example. Fig. 2(a) gives an energy level diagram of sample and STM tip. The tunneling transmission term $\mathrm{T}(\mathrm{E}, V)$ is shown, where $\mathrm{E}$ represents energy of electron and $V$ represent sample-tip bias voltage [11]. For $V>0$, the maximum of $\mathrm{T}(\mathrm{E}, V)$ occurs at the Fermi energy of the probe tip and $\mathrm{T}(\mathrm{E}, V)$ decreases when $\mathrm{E}$ decreases. For typical values of the average tunnel barrier of $4 \mathrm{eV}$ and sample-tip separation of 6 $\AA, T(E, V)$ changes by a factor of 20 as E varies over a $2 \mathrm{eV}$ interval [12]. As shown in Fig. 2(a), with large positive sample bias voltage ( $2.5 \mathrm{~V})$, all states contributing to tunneling current are well away from the band edge. Under this condition, then neglecting the effect of surface states and assuming for simplicity a trapezoidal barrier, the tunnel current can be expressed as

$$
I=C \times \exp \left\{-2 s \sqrt{2 m[\bar{\phi}-(e V / 2)] / \hbar^{2}}\right\}
$$

where $\bar{\phi}=\left(\phi_{t}+\xi+E_{C}-E_{F s}\right) / 2$ is the average tunnel barrier at zero bias [with symbols defined as in Fig. 2(a)] and $s$ is the tip-sample $\gtrsim$ separation. For large positive $V$, the constant $C$ in this equation is not dependent on $\quad V$, and furthermore, it is quite independent on the type of semiconductor into which the tunneling occurs [12]. This somewhat remarkable result arises from the fact that, as recognized in early studies of tunneling, the tunnel current is relatively independent of the density-of-states of the material [13]. This result has been confirmed by explicit calculations for a range of direct-gap III-V semiconductors [10], materials for which it is known that surface states for the (110) surface make only a small contribution to the tunneling characteristics [8]. The applicability of Eq. (1) means e.g. that in Fig. 2(a) the p-type material has less current (at fixed $s$ and $V$ ) than the $n$-type material not because the tunneling occurs at a different location in the conduction band, but rather, because the average barrier $\bar{\phi}-(e V / 2)$ is higher for the $p$-type material than for the $n$-type material.

Given the invariance of the constant $C$ in Eq. (1), it is clear how potentiometry can be performed. One scans the probe-tip maintaining a constant tip-sample 
separation $s$ (i.e. with the usual topographic feedback turned off) and then the sampletip bias voltage $V$ is adjusted to maintain a constant tunneling current $I$. In that case, $\bar{\phi}=$ constant so that

$$
\begin{aligned}
\Delta(e V) & =\Delta\left(\xi+E_{C}-E_{F s}\right) \\
& =\Delta \xi+\Delta E_{C}
\end{aligned}
$$

where Eq. (2b) is for the case of constant $E_{F s}$ across the junction (i.e. no applied bias across the junction). The quantity on the right-hand side of Eq. (2a) is simply the position of the vacuum level relative to the Fermi-level. Thus, the sample voltage yields a direct measure of the variation in vacuum level across the junction. The variation in vacuum level arises from some variation in electrostatic potential in the junction, e.g. for a $p n$-junction with this arises simply from the space-charge in the junction. If a bias is applied across the junction then the resulting variation in $E_{F s}$ will be reflected in both the vacuum level and the measured sample voltage. Figure 2(b) gives a schematic of basic experimental setup. In normal topography mode, $V_{\text {sample }}$ does not change through the scanning process. In potentiometry mode, a feedback circuit adjusts $V_{\text {sample }}$ to maintain a constant tunneling current $I$ while scanning the probe-tip at constant tip-sample separation. A very low drift rate in the STM is thus required in order to perform the measurement; in our instrument z-drift rates of less than $0.01 \AA / s$ are routinely obtained using drift compensation [14]. Atomically flat cleaves are also required for the measurement. A single monoatomic step will produce a change in the sample voltage of a few tenths of a volt, but larger steps could cause the potentiometry feedback loop to exceed its operating range leading to unintentional contact between the tip and sample.

It should be noted that the above discussion was presented for the case of a heterojunction, i.e. with different materials across the junction so that the possible variation of the parameter $C$ in Eq. (1) for the different materials is a consideration. However, for the case of a homojunction, the value of $\mathrm{C}$ will be identical on either side of the junction so the requirement of performing the measurement at relatively large, positive sample bias voltage is removed. In that case the effect of the potentiometric method can be seen as simply producing a constant alignment between the energy bands of the sample and probe-tip as the tip is scanned across the junction.

Figure 3(b) shows two potentiometry scans across the low-doped $p n$-junction, acquired with different starting voltages for the measurement. The left side of each scan corresponds to $p$-GaAs and the right side to $n$-GaAs, as can be seen in the image of Fig. 3(a) which was acquired from the same location as the potentiometric scans. The combined depletion width of $n$ - and $p$-type materials is expected to be $110 \mathrm{~nm}$ wide; the dashed lines in Fig. 3 show a computed potential profile across the depletion region (including the effects of the binding energies of Si donors and Be acceptors [15]), which is in good agreement with the observations. The total variation in potential across the junction is about $1.3 \mathrm{~V}$ for both the experiment and the theory. The experimental results of Fig. 3(b) are somewhat exceptional in this case; in more typical measurements we find the potential variation across the junction to be $1.0-$ $1.2 \mathrm{~V}$, that is, $0.1-0.3 \mathrm{~V}$ less than that expected theoretically. On the $n$-type side of the junction the potential curves in Fig. 3(b) displays some peaks due to discrete dopant atoms and residual surface defects and/or contamination. As seen in Fig. 3(a) those surface defects or contaminants give rise to variations in the surface height, which in turn induce a significant change in the tunnel current so that a substantial variation in the sample voltage is needed to maintain a constant current. Such effects 
could be eliminated, if desired, by averaging of potentiometric scans acquired over consecutive surface locations.

Results across the highly-doped $p n$-junction are shown in Fig. 4, with Fig. 4(a) showing a constant-current STM image and Fig. 4(b) displaying a potential scan across this region. The proximity of the AlAs layer located $50 \mathrm{~nm}$ away from this highly-doped $p n$-junction has a substantial influence on the results. The cleavage quality at the AlAs layer is generally not good, as seen in Fig. 1, so that surface steps and other defects were present there in all of our attempts at cleaving the wafer. Therefore we found that the surface Fermi-level was located near midgap at the AlAs layer, and consequently the potentiometric scans showed a large increase as one approaches the AlAs layer from the neighboring $n^{++}$region. The data at the left-hand side of Fig. 4(b) shows the start of this increase of the potential. Nevertheless, further to the right in Fig. 4(b) the effects of the AlAs layer are negligible, and we observe the expected behavior for this highly-doped $p n$-junction. The combined depletion width of $n$-type and $p$-type regions is $35 \mathrm{~nm}$ in this case, as shown by the dashed line in Fig. 4(b).

\section{Discussion}

The potentiometric method proposed here can be compared with that used in prior studies [1-6]. For metals, potentiometry is conventionally performed by searching for the sample-tip bias voltage at which the tunnel current is zero [3]. As a function of probe-tip position, this bias provides an exact measure of any variation in electrostatic potential in the sample. Our new method described above is similar, except that rather than searching for a zero current point we search, with fixed $s$, for some fixed non-zero current value. The method is thus applicable to both metals and semiconductors, with the condition that for a semiconductor heterojunction the tunneling must occur well up into the conduction band.

Our method is complementary to that of spatially resolved spectroscopy and subsequent analysis of the $I(V)$ curves to yield a band edge position [6]. To illustrate the difference between those two techniques, consider scanning across a heterojunction with conduction band offset $\Delta E_{C}$. Variation in the spatially resolved $I(V)$ curves should yield a direct measure of $\Delta E_{C}$. On the other hand, our potentiometric measurement will yield $\Delta \xi+\Delta E_{C}$ [from Eq. (2b)]. In the usual simplest model for heterojunctions, one expects $\Delta E_{C}=-\Delta \xi$ [16], so that our method would produce zero change across the heterojunction. This result is consistent with a zero change in electrostatic potential across the junction. If $\Delta E_{C}$ differs from $-\Delta \xi$, their difference is sometimes referred to as a microscopic interface dipole [16]. Our method will thus measure only this microscopic dipole term, and in this sense it is quite different from the prior technique based on spatially resolved spectroscopy [6]. Similarly, the original method of Muralt et al. [1,2] involves a large modulation in the voltage such that $I(V)$ curves extending across the band gap are measured. This measurement will thus also be sensitive to band edge variations in the tunnel current, so that for a heterostructure one would not obtain simply the variation in electrostatic potential, but rather, something which also involves the conduction band offset across the junction.

As discussed in the prior section, one requirement to achieve quantitative potentiometric results with our method is that the sample Fermi-level at the cleavage 
surface not be constrained (i.e. pinned) by any electronic states at the surface. Another important consideration is that of tip-induced band bending. As remarked in Section II our measurements were performed under laser illumination of the cleavage face, but in fact this illumination was found to have a negligible effect on the results. In Fig. 3(b) we find good agreement between the experimental and theoretical potential variations, although as discussed there we find a typical error of $0.1-0.3 \mathrm{~V}$ between experimental and theoretical results for that junction. We compare this value with that expected theoretically for band bending due to a sharp tip in proximity to a semiconductor [8], as shown in Fig. 5. We first consider a paraboloid-shaped probetip. We compute the surface band bending for a $+3 \mathrm{~V}$ voltage bias on the tip relative to flat band conditions in the semiconductor [17], for $n$-type or $p$-type material with doping density $1 \times 10^{18} \mathrm{~cm}^{-3}$. From Section II we expect tip radii in the range $20-40$ $\mathrm{nm}$, which from Fig. 5 would imply a difference in tip-induced band bending between $n$ - and $p$-type material of about $0.7 \mathrm{eV}$ based on the theory, significantly greater than that observed experimentally. We have also considered the effect of roughness on the probe-tip apex. As in our past work [8], we place a $1 \mathrm{~nm}$ radius hemisphere at the end of the paraboloid (maintaining a fixed separation of $1 \mathrm{~nm}$ between the tip apex and the sample). The theoretical band bending results are then reduced; for $n$-type material the reduction, for tip radii of $20-40 \mathrm{~nm}$, is about $0.30 \mathrm{eV}$ whereas for $p$-type material the reduction is only $0.07 \mathrm{eV}$. Thus, on the basis of the theory we expect a difference in band bending between $n$ - and $p$-type material of about $0.5 \mathrm{eV}$, significantly greater than our observed difference between experiment and theory of $0.1-0.3 \mathrm{~V}$.

We conclude that, in terms of tip-induced band bending, the potentiometric method used here yields results that are somewhat more accurate (i.e. less affected by tip-induced band bending) than one might expect on the basis of simple electrostatic band bending computations. In any case, it is clear from Fig. 5 that the 3D nature of the probe-tip plays a very important role in reducing tip-induced band bending (for the parameters of Fig. 5, the band bending for infinite tip radius is $1.53 \mathrm{eV}$ and 0.30 $\mathrm{eV}$ for $n$ - and $p$-type material, respectively). The theory indicates that tip-induced band bending is not negligible, but our experiments show that it actually has nearly the same value for $n$ - and $p$-type material. A complete understanding of this situation requires additional work.

Regarding the spatial resolution of the potentiometric method proposed here, as shown in Fig. 4(b) we can clearly resolve potential variations at length scales of less than $10 \mathrm{~nm}$. Similar results have been obtained for all the other potentiometric methods based on tunnel current [1-6]. In fact, one expects in most cases that the observed spatial variations in the potential will be limited more by the depletion widths in the semiconductors than by intrinsic resolution of the methods themselves, since their ultimate resolution should be on the atomic-scale as for any other type of STM-based measurement. Comparing our potentiometric method with the previous ones [1-6], we believe that the most significant advantage of our method is the fact that the raw data itself directly provides the measure of the electrostatic potential. For the case of the spatially resolved $I(V)$ measurements some analysis is needed to extract the variation in potential [6], and for other types of measurements it is even more difficult to extract the potential. A disadvantage of our method is the fact that it does require an atomically flat cleavage surface. In addition, during the measurement the tip is scanned across the surface with the topographic feedback turned off, thereby requiring an effective means of nulling out any relative angle between the tip motion 
and the sample surface as well as eliminating any relative drift in their vertical positions. Finally, we note that all potentiometric measurements, if performed on rough surfaces, can suffer from significant problems of tip-sample convolution as discussed by Pelz et al. [18]

Concerning the above mentioned requirement for an atomically flat cleavage surface in order to apply our method, this requirement can be relaxed if a means is found to leave the usual topographic feedback on during the measurement. This can be accomplished, at least for measurements of small variations in surface potential, using the following technique. Consider acquiring a constant-current topographic line scan $\delta s$ across a surface, carefully leveling the scan so that $\delta s=0$ where no potential variation occurs. Then one modulates both the sample tip separation $s$ and the sampletip voltage $V$, obtaining $\mathrm{d} I / \mathrm{d} s$ with $V$ constant and $\mathrm{d} I / \mathrm{d} V$ with $s$ constant using two lock-in amplifiers. From those data the variation in potential can be obtained from $\delta V$ $=\delta s(\mathrm{~d} I / \mathrm{d} s) /(\mathrm{d} I / \mathrm{d} V)$.

\section{Conclusion}

In conclusion we propose a new STM based potentiometry method based on the fact that the tunnel current, in the absence of surface states and to a good approximation, depends only on the characteristics of the tunnel barrier. The method yields a direct measure of the potential variation across e.g. III-V semiconductor junctions viewed on a (110) face for which surface state effects are known to be relatively small. Illustration of this technique has been accomplished on a GaAs pnjunction, with the experimental results being in good agreement with the theoretical potential distribution.

\section{Acknowledgements}

We thank Ashutosh Sagar for his assistance in constructing the feedback circuit used here. Discussions with B. Grandidier and C. Van de Walle are gratefully acknowledged. This work was supported by the National Science Foundation, grant DMR-9985898.

\section{References:}

[1] P. Muralt and D. W. Pohl, Appl. Phys. Lett. 48, 514 (1986).

[2] P. Muralt, H. Meier, D. W. Pohl, and H. W. M. Salemink, Appl. Phys. Lett. 50, 1352 (1987).

[3] J. R. Kirtley, S. Washburn, and M. J. Brady, Phys. Rev. Lett. 60, 1546 (1988).

[4] S. Kordic, E. J. van Loenen, and A. J. Walker, Appl. Phys. Lett. 59, 3154 (1991).

[5] S. Hosaka, S. Hosoki, K. Takata, K. Horiuchi, and N. Natsuaki, Appl. Phys. Lett. 53, 487 (1988).

[6] E. T. Yu, M. B. Johnson, and J.-M. Halbout, Appl. Phys. Lett. 61, 201 (1992).

[7] R. Gomer, Field Emission and Field Ionization (Harvard University Press, Cambridge, 1961), p. 19 and 47.

[8] R. M. Feenstra, Phys. Rev. B 50, 4561 (1994).

[9] R. M. Feenstra, E. T. Yu, J. M. Woodall, P. D. Kirchner, C. L. Lin, and G. D. Pettit, Appl. Phys. Lett. 61, 795 (1992).

[10] A. Vaterlaus, R. M. Feenstra, P. D. Kirchner, J. M. Woodall, and G. D. Pettit, J. Vac. Sci. Technol. B 11, 1502 (1993).

[11] R. M. Feenstra, J. A. Stroscio, and A. P. Fein, Surf. Sci. 181, 295 (1987).

[12] R. M. Feenstra, Physica B 273-274, 796 (1999). 
[13] C. B. Duke, Tunneling in Solids (Academic, New York, 1969), Eq. (7.8b) and discussion following.

[14] R. M. Feenstra and M. A. Lutz, J. Vac. Sci. Technol. B 9, 716 (1991).

[15] Using computed room temperature Fermi-level positions of $E_{\mathrm{C}}-E_{\mathrm{Fs}}=25 \mathrm{meV}$ and $E_{\mathrm{Fs}}-E_{\mathrm{V}}=95 \mathrm{meV}$ for $\mathrm{Si}$-doped $n$-type material and Be-doped $p$-type material respectively, we find $1.30 \mathrm{eV}$ separation of the Fermi-level positions between $n$ and $p$-type materials.

[16] A. Franciosi and C. G. Van de Walle, Surf. Sci. Rep. 25, 1 (1996).

[17] We choose a constant offset bias relative to flat band conditions in order to simulate the potentiometry measurement. Flat band conditions occur when $\phi_{t}=$ $\xi+E_{C}-E_{F s}-V$, so the $V=\xi+E_{C}-E_{F s}-\phi_{t}$. For the parameters used in this work and with $\xi=4.1 \mathrm{eV}$ and $\phi_{t}=5 \mathrm{eV}$ this corresponds to sample voltages of $V=-0.9$ $\mathrm{V}$ and $+0.4 \mathrm{~V}$ for $n$ - and $p$-type material, respectively. Thus, a $+3 \mathrm{~V}$ offset then corresponds to actual sample biases of $+2.1 \mathrm{~V}$ and $+3.4 \mathrm{~V}$ for $n$ - and $p$-type material, respectively.

[18] J. P. Pelz and R. H. Koch, Phys. Rev. B 41, 1212 (1990).

(a) (001) growth direction $\longleftarrow \quad \longmapsto 50 \mathrm{~nm}$
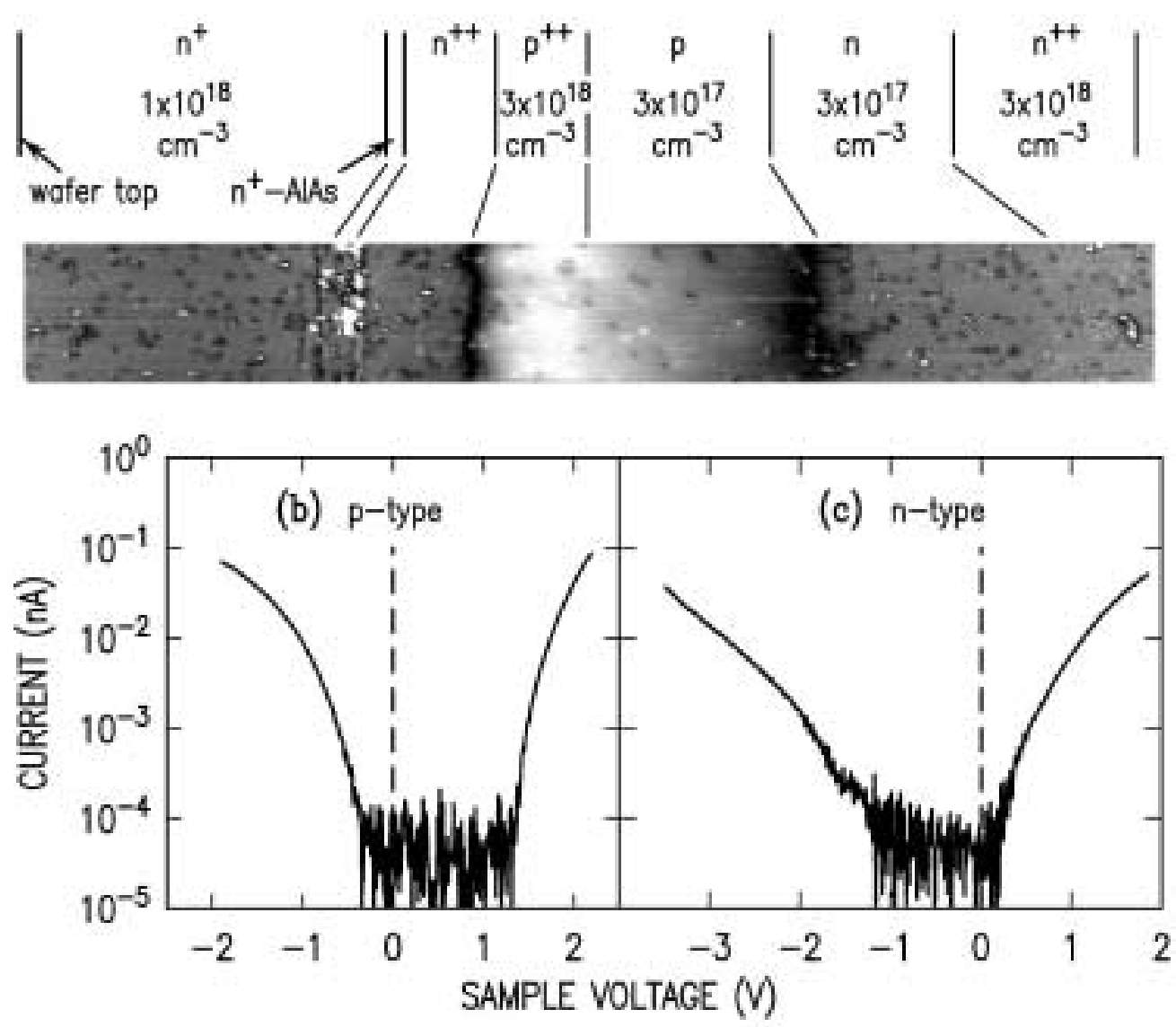

Fig. 1 (a) Large-scale constant-current STM topograph of $\operatorname{GaAs}(1 \overline{1} 0)$ surface, acquired with sample voltage of $-2.0 \mathrm{~V}$. Above the image the grown heterostructure is indicated. (b) and (c) Typical current-voltage curves acquired on $p$-type and $n$-type GaAs layers $\left(3 \times 10^{17} \mathrm{~cm}^{-3}\right.$ doping concentration) respectively. The sample voltage corresponds to the energy of a state relative to the Fermi-level $(0 \mathrm{~V})$. 


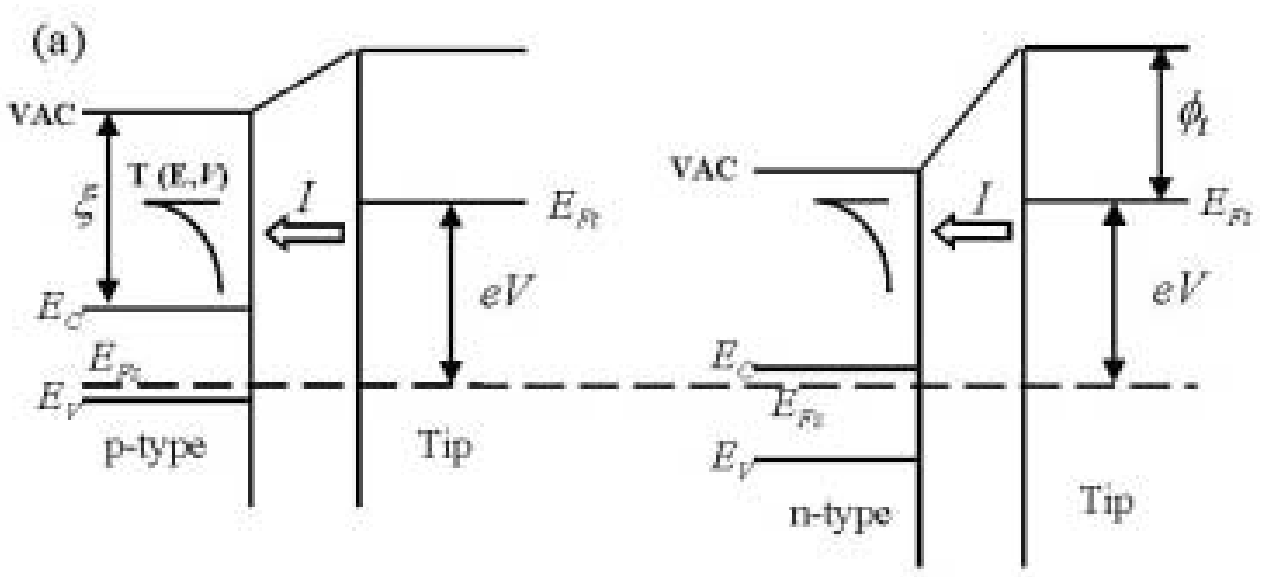

(b)

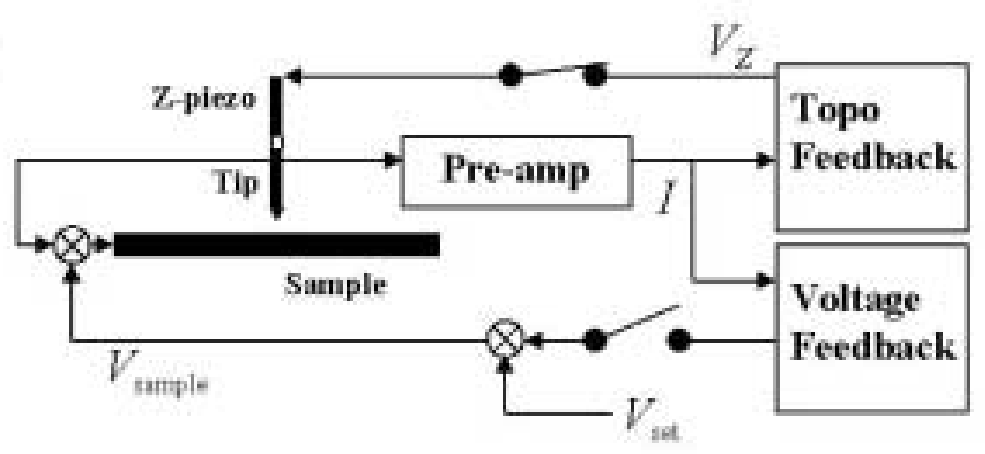

Fig. 2 (a) Energy level diagrams of STM probe-tip and sample, with $p$-type and $n$ type samples shown separately. In both cases, a large positive sample voltage has been applied. The tunneling transmission term $\mathrm{T}(\mathrm{E}, V)$ are indicated, showing all sample states contributing to tunneling current are well inside the conduction band. (b) Schematic of experimental setup. In topography mode, $V_{\text {sample }}$ equals a specified value, $V_{\text {set }}$, and the topographic feedback is active. In potentiometry mode, the topographic feedback is disabled and a separate feedback circuit adjusts $V_{\text {sample }}$ to maintain a constant tunneling current $I$ while the probe-tip is scanned with constant sample-tip separation (the starting value of $V_{\text {sample }}$ is given by $V_{\text {set }}$ ). 

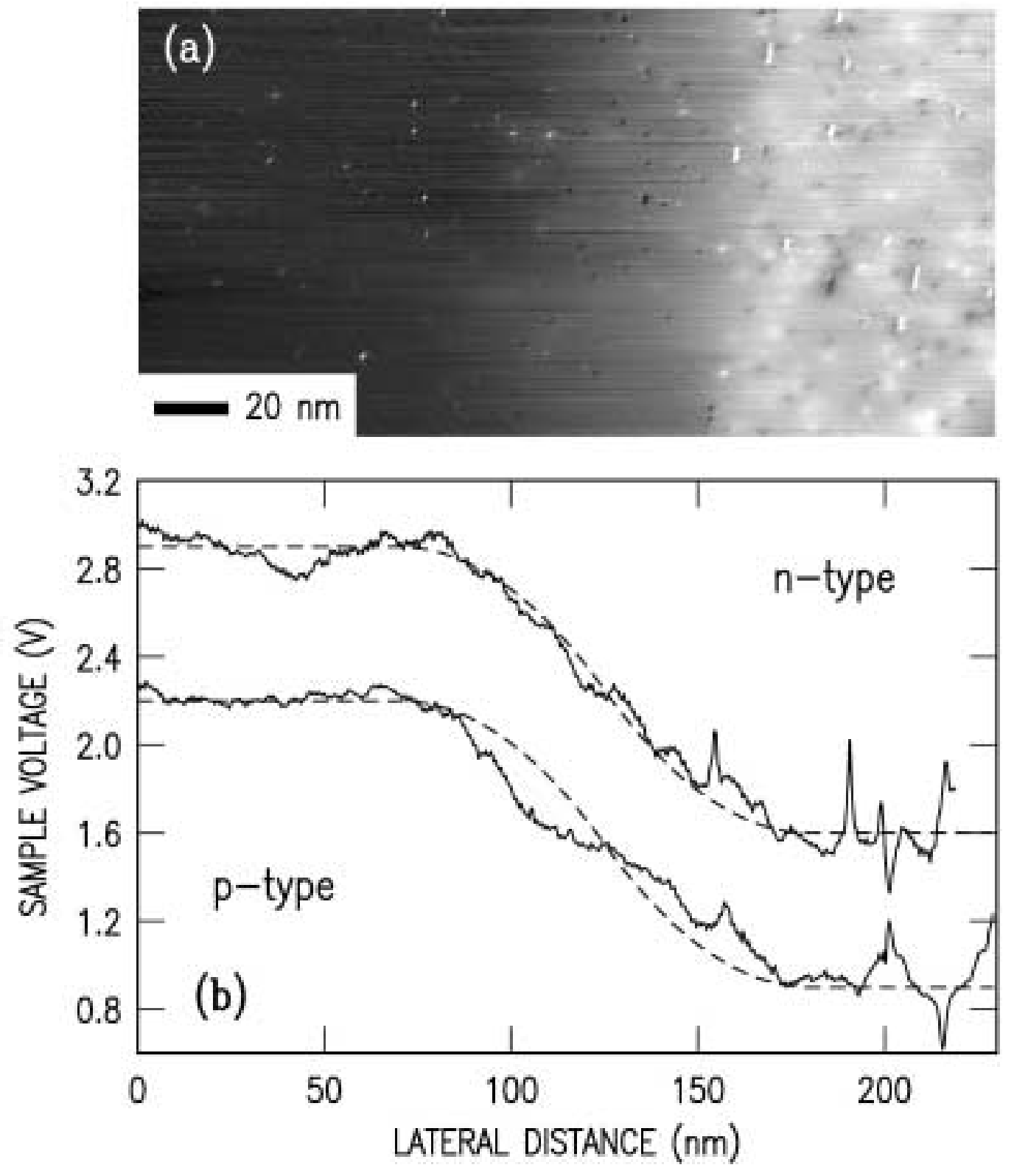

Fig. 3 (a) STM topograph of $3 \times 10^{17} \mathrm{~cm}^{-3}$ doped $p n$-junction, acquired with sample voltage of $3.0 \mathrm{~V}$. (b) Two potentiometry scans across the junction, acquired with different starting voltages for the scans. Dashed curves show the theoretical potential distribution for this $p n$-junction. 


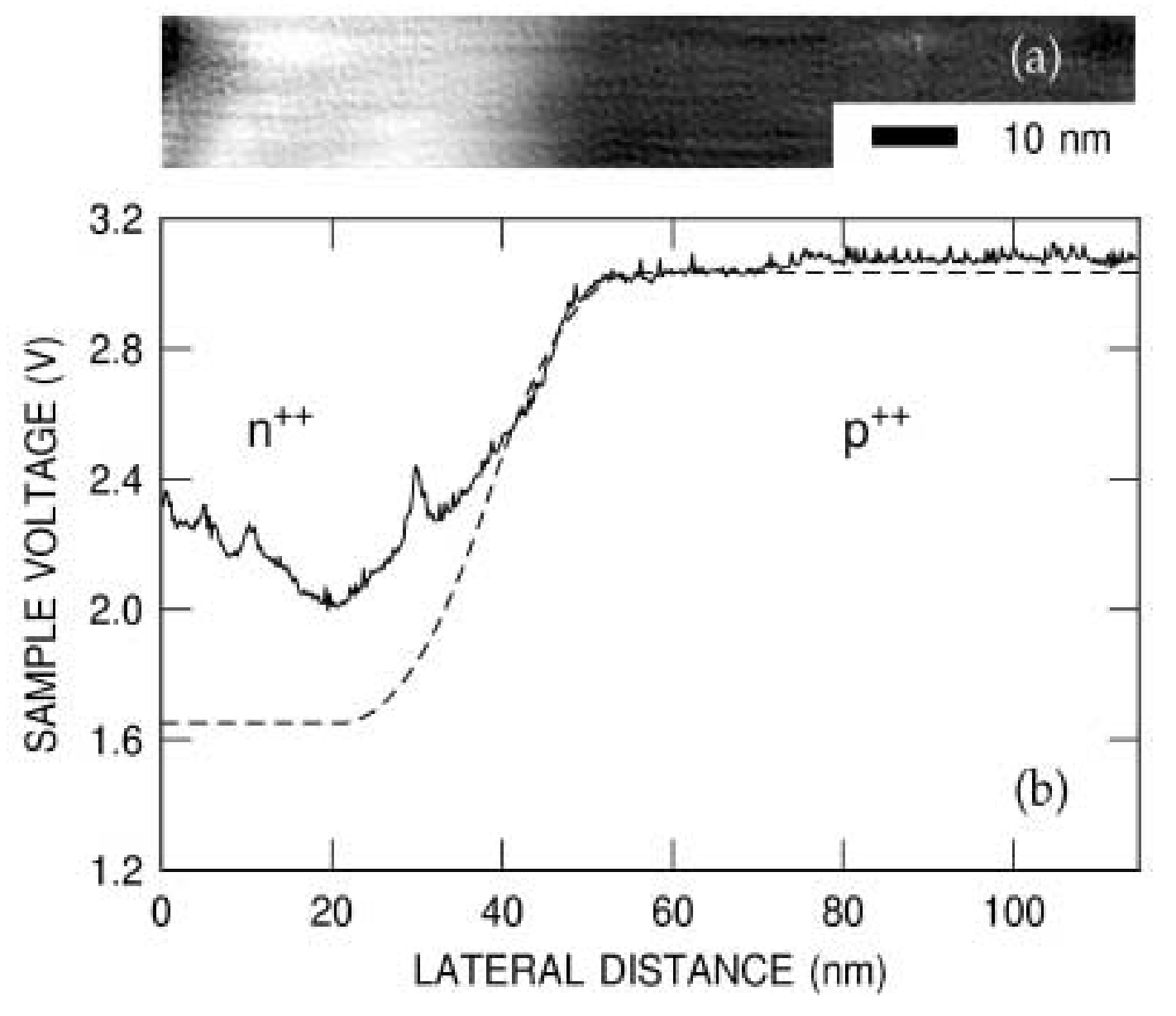

Fig. 4 (a) STM topograph of $3 \times 10^{18} \mathrm{~cm}^{-3}$ doped $p n$-junction, acquired with sample voltage of $2.5 \mathrm{~V}$. (b) Representative potentiometry scan across the junction. Dashed curve shows the theoretical potential distribution for this $p n$-junction. 


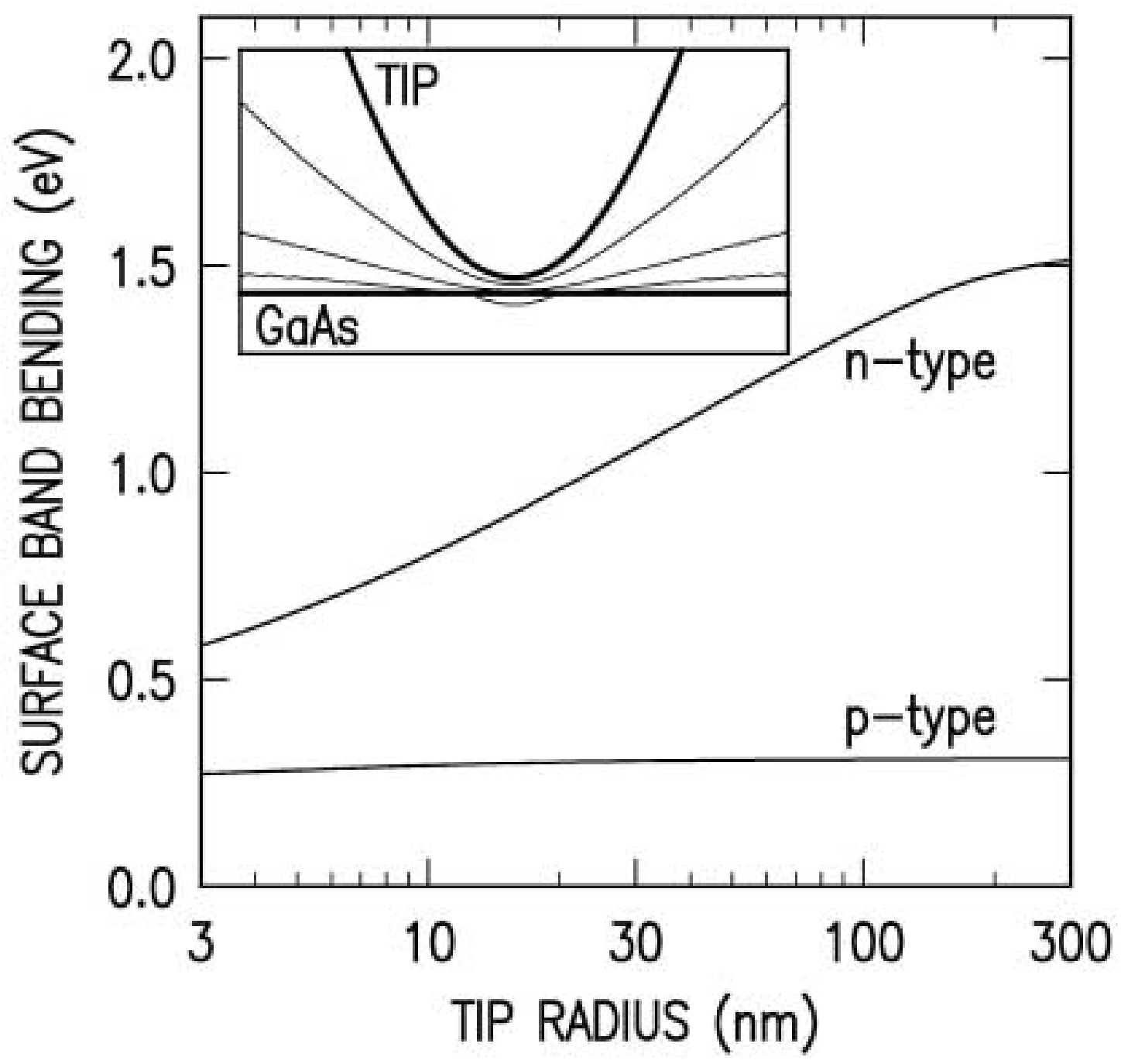

Fig. 5 Computed surface band bending results, for $n$ - and $p$-type GaAs with doping density of $1 \times 10^{18} \mathrm{~cm}^{-3}$. A paraboloid-shaped probe-tip is used, with $1 \mathrm{~nm}$ separation between the tip apex and the sample. The sample has a $+3 \mathrm{~V}$ offset in voltage relative to flat band conditions in the semiconductor, so that for the $n$-type results the semiconductor surface is in depletion and for the $p$-type results it is in accumulation. The computed surface band bending at a point directly below the tip apex is plotted, as a function of the radius of curvature of the probe-tip. The inset shows a tip with 4 $\mathrm{nm}$ radius in proximity to $n$-GaAs, with the thin lines showing equipotential contours corresponding to $0.5,1.0$, and $2.0 \mathrm{~V}$. 